\title{
GASTROPLASTIA VERTICAL COM BANDAGEM EM Y-DE-ROUX: ANÁLISE DE RESULTADOS
}

RESULTS OF ROUX-EN-Y VERTICAL BANDED GASTROPLASTY

\author{
Antonio Carlos Valezi, TCBC-PR ${ }^{1}$ \\ Jorge Mali Júnior ${ }^{2}$ \\ Edivaldo Macedo de Brito, ACBC-PR ${ }^{3}$ \\ Antonio César Marson, ACBC-PR ${ }^{4}$
}

\begin{abstract}
RESUMO: Objetivo: Avaliar prospectivamente os resultados de pacientes obesos mórbidos submetidos a gastroplastia vertical com bandagem em Y-de-Roux no Hospital Universitário da Universidade Estadual de Londrina. Método: Analisamos consecutivamente 250 pacientes, sem cirurgia bariátrica prévia, que foram submetidos à cirurgia que associa a gastroplastia vertical com bandagem e derivação gastrojejunal em Y-deRoux. Os parâmetros utilizados para análise dos resultados foram, morbidade, mortalidade e redução ponderal. O seguimento dos pacientes foi de, no mínimo, um ano. O índice de massa corpórea médio foi de $46 \mathrm{Kg} / \mathrm{m}^{2}$. Resultados: A incidência de complicações operatórias que exigiram reoperação foi de $2 \%$. Não houve óbito na presente série. Após um a três anos de "follow-up", observamos uma redução do peso pré-operatório em média de 37,5\%. Acompanhando a perda de peso, detectamos uma importante melhora nas comorbidades, e, em alguns casos, controle total da doença associada com a obesidade. Conclusões: A gastroplastia vertical com bandagem em Y-de-Roux foi,em nosso serviço, efetiva em produzir perda de peso intensa e duradoura associada à baixa taxa de morbi-mortalidade.
\end{abstract}

Descritores: Gastroplastia; Obesidade mórbida.

\section{INTRODUÇÃO}

A obesidade é atualmente um dos mais graves problemas de saúde pública, com proporções epidêmicas na Europa e nos Estados Unidos (EUA). Nos EUA, a obesidade é considerada a segunda causa evitável de morte, com 300.000 mortes / ano, sendo a primeira o tabagismo ${ }^{1,2}$.

No Brasil, segundo estimativas do IBGE, a incidência de obesidade é de 60 milhões de pessoas, das quais $20 \%$ são crianças.

A obesidade, além de problemas psicológicos e sociais que acarreta, também determina diversos problemas médicos como o aparecimento de inúme- ras doenças crônicas, mais frequentemente diabetes mellitus, hipertensão arterial sistêmica, doenças osteoarticulares, disfunções pulmonares, responsáveis, não só por impacto negativo na qualidade de vida, como elevação da morbi-mortalidade verificada nesses doentes ${ }^{3}$.

Dentre os obesos, destacam-se os obesos mórbidos, cujo impacto da doença faz-se sentir de maneira mais intensa.

Visando o tratamento desse grupo em particular, surgiram técnicas cirúrgicas, já que os tratamentos conservadores à base de dietas e drogas são incapazes de produzir perdas de peso significativas e duradouras, com índices de recidiva elevados ${ }^{4}$.

1. Professor Adjunto Doutor do Departamento de Cirurgia da Universidade Estadual de Londrina.

2. Médico Residente de Cirurgia Geral da Universidade Estadual de Londrina.

3. Professor Adjunto Mestre do Departamento de Cirurgia da Universidade Estadual de Londrina.

4. Professor Assistente Mestre do Departamento de Cirurgia da Universidade Estadual de Londrina.

Recebido em 12/04/2003

Aceito para publicação em 21/11/2003

Trabalho Realizado na Disciplina de Cirurgia do Aparelho Digestivo do Departamento de Clínica Cirúrgica da Universidade Estadual de Londrina. 
A cirurgia considerada padrão ouro para esse tipo de doente é a gastroplastia vertical com bandagem em Y-de-Roux (GVBYR), proposta por Fobi $^{5}$ e Capella ${ }^{6}$.

O objetivo deste trabalho é relatar a experiência do Serviço de Cirurgia do Aparelho Digestivo do Hospital Universitário Regional do Norte do Paraná (HURNP) da Universidade Estadual de Londrina (UEL), no tratamento cirúrgico da obesidade mórbida.

\section{MÉTODO}

Foram analisados prospectivamente $250 \mathrm{pa}-$ cientes obesos mórbidos, sem cirurgia bariátrica prévia, submetidos à gastroplastia vertical com bandagem em Y-de-Roux ( Cirurgia de Fobi-Capella) no HURNP da UEL, no período de Janeiro de 1999 à dezembro de 2001.

Dos 250 pacientes estudados, $189(75,6 \%)$ pertenciam ao sexo feminino e $61(24,4 \%)$ ao masculino. A média de idade foi de 41 anos (17 - 64 anos). A altura média foi de $1,69 \mathrm{~m}$ (1,55 a 1,89 m). O peso pré-operatório situou-se entre 88 e $260 \mathrm{Kg}$, com média de $146 \mathrm{Kg}$. O índice de massa corpórea (IMC) médio foi de $46 \mathrm{Kg} / \mathrm{m}^{2}\left(36\right.$ a $\left.79 \mathrm{Kg} / \mathrm{m}^{2}\right)$.

Na Tabela 1, encontram-se as doenças associadas ou decorrentes do excesso de peso detectados na população em estudo.

Tabela 1 - Doenças associadas em 250 obesos mórbidos operados.

\begin{tabular}{lcc}
\hline Doenças & n & \% \\
\hline Osteoartropatias & 63 & 25,2 \\
Hipertensão Arterial & 52 & 20,8 \\
Diabetes Mellitus & 44 & 17,6 \\
Hérnia de Hiato & 35 & 14,0 \\
Varizes & 31 & 12,4 \\
Hipercolesterolemia & 29 & 11,6 \\
Dispnéia & 28 & 11,2 \\
Colelitíase & 17 & 6,8 \\
Apnéia do Sono & 14 & 5,6 \\
Dermatopatia & 09 & 3,6 \\
Broncoespasmo & 09 & 3,6 \\
Amenorréia & 08 & 3,2 \\
Arritmia & 06 & 2,4 \\
Sem doenças associadas & 115 & 46,0 \\
Mais de duas doenças & 18 & 7,2 \\
\hline
\end{tabular}

Os pacientes foram inicialmente submetidos a uma avaliação clínica realizada por uma equipe multidisciplinar composta por: cirurgião, endocrinologista, psicólogo e nutricionista. Durante a anamnese clínica, os pacientes eram questionados sobre alguns aspectos da obesidade: início do quadro, tipos de tratamento já realizado, presença de comorbidades, antecedentes pessoais e familiares, e hábitos alimentares. Calculava-se o grau de obesidade através do IMC (peso $(\mathrm{Kg})$ dividido pela altura $\left(\mathrm{m}^{2}\right)$ ).

O consentimento informado era obtido de todos os pacientes, após orientações a respeito dos motivos de indicação cirúrgica, a técnica a ser realizada, exames pré-operatórios necessários, mudanças de hábitos alimentares após a cirurgia, riscos inerentes à cirurgia e necessidade de seguimento pós-operatório prolongado.

Os exames pré-operatórios solicitados foram: hemograma e coagulograma completos, glicemia, creatinina, transaminases, eletroforese de proteínas, hormônios tireoidianos, lipidograma, acido úrico, ferro, urina I, parasitológico de fezes, radiografia de tórax, avaliação de função pulmonar, eletrocardiograma, ultra-sonografia abdominal, endoscopia digestiva alta e pesquisa de $H$. pylori, entre outros eventualmente necessários.

Os critérios de exclusão para o tratamento cirúrgico foram: presença de pneumopatias graves, distúrbios psiquiátricos e/ou psicológicos, cardiopatias graves, dependência de drogas ilícitas e álcool, e insuficiência renal.

Os doentes selecionados, para a realização do tratamento cirúrgico, obedeceram alguns critérios expostos na Tabela 2.

Todos os pacientes candidatos ao tratamento cirúrgico eram encaminhados para consulta psicológica e/ou psiquiátrica, para avaliação da estrutura de sua personalidade para enfrentar as mudanças biopsicossociais decorrentes da cirurgia. Durante a entrevista, todos os pacientes relatavam dificuldade de integração e convívio social.

Os pacientes submetiam-se a jejum de oito horas antes da cirurgia, recebiam medicação préanestésica uma hora antes de serem encaminhados ao centro cirúrgico, além de antibióticos e heparinização para prevenção de infecções e fenômenos tromboembólicos.

$\mathrm{O}$ ato cirúrgico iniciava-se com uma incisão mediana supra-umbilical. Seccionamos a alça jejunal a $50 \mathrm{~cm}$ da transição duodeno-jejunal. Sepultamos o coto jejunal distal em dois planos de sutura. Constru- 
Tabela 2 - Critérios de seleção para o tratamento cirúrgico.

\begin{tabular}{|l|}
\hline 1- $\mathrm{IMC}=40 \mathrm{Kg} / \mathrm{m}^{2}$. \\
\hline 2- $\mathrm{IMC}=35 \mathrm{~kg} / \mathrm{M} 2$ associado a doenças agravadas pela obesidade. \\
\hline 3- Risco cirúrgico aceitável. \\
\hline 4- Capacidade do paciente compreender as implicações da operação. \\
\hline 5- Tentativa de emagrecimento por medidas clínicas. \\
\hline
\end{tabular}

ímos uma alça em Y-de-Roux de $100 \mathrm{~cm}$ através de enteroenteroanastomose término-lateral em dois planos de sutura.

A septação gástrica era realizada com disparos sucessivos de grampeador linear cortante 75 $\mathrm{mm}$, carga verde, criando-se um reservatório gástrico vertical com capacidade em torno de $50 \mathrm{ml}$ junto à cárdia, de $10 \mathrm{~cm}$ ao longo da pequena curvatura gástrica. Reforçamos a linha de sutura de grampos do estômago excluído com chuleio simples de poliglactina 3.0. Realizamos duas suturas, uma hemostática e outra invaginante, no reservatório gástrico com chuleio simples de poliglactina 3.0.

Testamos a integridade das suturas injetando azul de metileno diluído através da sonda de Fouchet. Envolvemos a pequena bolsa gástrica, a $2 \mathrm{~cm}$ de sua extremidade distal, com anel de silicone de $6,4 \mathrm{~cm}$ de circunferência.

A alça de Roux é passada através de brecha feita no mesocolon transverso para o andar supramesocólico. Realizamos gastroenteroanastomose término-lateral em dois planos de sutura e testamos sua integridade com azul de metileno.

Procedíamos então ao fechamento da cavidade abdominal, por planos, utilizando para aponeurose prolene 2 com sutura contínua interrompida. Aproximamos o tecido celular subcutâneo com catgut simples 2.0 e deixamos um dreno de sucção exteriorizado por contra-abertura. A síntese da pele era feita com pontos separados de nylon 3.0 ou 4.0.

Após a cirurgia todos os pacientes eram encaminhados para UTI e mantidos lá por pelo menos 12 horas. Raramente os doentes eram encaminhados intubados à UTI. Como profilaxia de acidentes tromboembólicos, mantínhamos heparina de baixo peso molecular até o $10^{\circ}$ dia de pós-operatório e orientávamos deambulação precoce. No pós-operatório imediato, os doentes ficavam em jejum e recebiam hidratação parenteral com suporte calórico e hidroeletrolítico adequados. No $2^{\circ}$ dia de pós-operatório eram ofertados líquidos com baixos teores de calorias, tais como: água, chá e suco de frutas. A dieta líquida hipocalórica era mantida durante o primeiro mês.

Geralmente entre o $4^{\circ}$ e $7^{\circ}$ dia após a operação os pacientes recebiam alta hospitalar, e eram encaminhados para seguimento ambulatorial. Após a $4^{\mathrm{a}}$ semana progredia-se para uma dieta geral fracionada, excluindo-se líquidos e pastosos ricos em calorias.

Orientamos seguimento ambulatorial pós-operatório mensalmente durante o primeiro ano e a cada seis meses subseqüentemente. A cada retorno, os pacientes tinham seu peso aferido e eram questionados sobre a alimentação, além da presença de qualquer sintoma como, vômitos, fadiga e câimbras, no intuito de se diagnosticar precocemente qualquer deficiência nutricional ou complicação pós-operatória. Nessas consultas ambulatoriais também eram adaptados os tratamentos para as doenças associadas à obesidade em regressão.

A análise da evolução ponderal pós-operatória durante o seguimento ambulatorial era realizada através do peso corporal aferido e o cálculo do IMC, comparados com os valores pré-operatórios.

\section{RESULTADOS}

A morbidade pós-operatória imediata relacionou-se principalmente com problemas de pequena gravidade, como infecção de incisão cirúrgica, coleções serosas na incisão e infecções do trato urinário, entre outras de maior gravidade resumidas na Tabela 3.

Excetuando-se as complicações de pequena gravidade, e atendo-nos às mais graves, a nossa experiência mostra que houve 23 complicações imediatas no total de 250 cirurgias: complicações respiratórias em 11 pacientes, dos quais em dois casos a causa foi embolia pulmonar; lesão esplênica em 10 pacien- 
Tabela 3 - Complicações imediatas em 250 casos de GVBYR.

\begin{tabular}{lcr}
\hline Complicações & n & \% \\
\hline Serosidade na incisão & 03 & 7,3 \\
Lesão esplênica & 10 & 24,4 \\
Pneumonia & 09 & 21,9 \\
Supuração na incisão & 03 & 7,3 \\
Infecção urinária & 12 & 29,3 \\
Embolia pulmonar & 02 & 4,9 \\
Coleção Intracavitária & 02 & 4,9 \\
\hline Total & 41 & 100,0 \\
\hline
\end{tabular}

tes, que foram tratadas com esplenectomia em seis casos e esplenorrafia em quatro doentes.

As complicações pós-operatórias tardias são menos freqüentes e de menor gravidade, e ocorreram em 16 casos. Quadros de obstrução intestinal por bridas foram por nós registrados em dois casos, que exigiram correção cirúrgica. Úlceras, na anastomose gastrojejunal, ocorreram em 10 casos (4\%), com diagnóstico feito por endoscopia digestiva alta (EDA) e tratamento com bloqueadores de secreção gástrica. Houve cinco casos (2\%) de hemorragia digestiva alta, um por lesão aguda de mucosa gástrica, um caso de sangramento na anastomose gastrojejunal, e outros três devido à úlcera de boca anastomótica. Todos os cinco tratados clinicamente.

Complicações relacionadas ao anel de silicone ocorreram em três casos (1,2\%): em dois casos houve migração do anel distalmente ao nível da anastomose gastrojejunal, que causou obstrução do trânsito intestinal, tendo sido necessária a sua retirada por laparotomia; em um caso houve extrusão do anel para a luz da bolsa gástrica, que foi retirado por EDA.

As complicações nutricionais se fizeram presente nesta série em 71 casos (28,4\%): 68 doentes com anemia ferropriva; dois casos de deficiência de vitamina $\mathrm{B}$ e um doente com desnutrição proteicocalórica leve. Todos foram tratados com reposição de sais minerais, vitaminas e ajustes na alimentação.

Trinta e um doentes $(12,4 \%)$ desenvolveram colelitíase e foram submetidos a colecistectomia .

Houve necessidade de cinco reoperações (2\%), quatro já citadas acima, e outra por abscesso subfrênico, no $7^{\circ}$ pós-operatório. Houve outro caso de coleção intracavitária tratada conservadoramente com antibióticos e punção percutânea guiada por tomografia computadorizada de abdome.

Hérnias incisionais ocorreram em 16 pacientes $(6,4 \%)$.

A Figura 1 mostra a média de perda de peso avaliados nos momentos pré-operatório e nos primeiros 12 meses de seguimento. Nos 250 pacientes operados, a média de perda de peso após o primeiro ano de seguimento foi de $37,5 \%$ do peso pré-operatório.

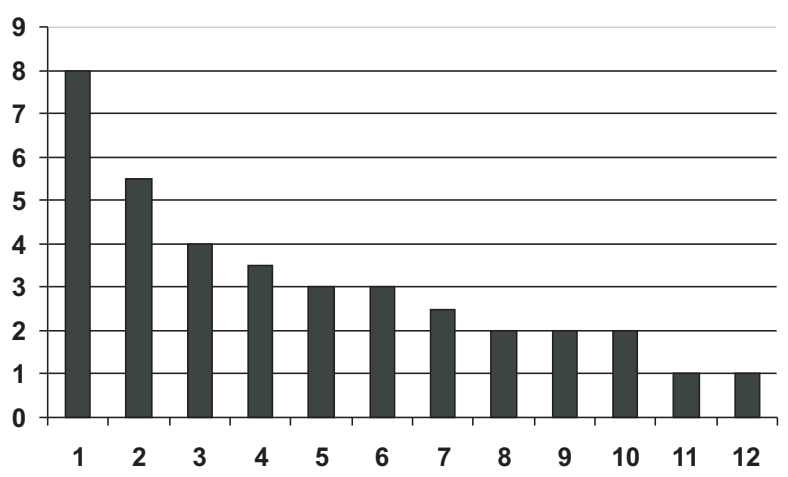

Figura 1 - Percentual de perda de peso nos primeiros 12 meses após GVBYR.

O seguimento ambulatorial tardio mostrou também que, acompanhando a redução ponderal, houve grande melhora das comorbidades na maioria dos casos, como ilustra a Tabela 4.

Tabela 4 - Relação do emagrecimento pós-cirurgia com a doença associada.

\begin{tabular}{llccc}
\hline Doenças & n & Melhora & Cura & Inalterada \\
\hline Diabetes & 44 & 12 & 32 & - \\
Artropatia & 63 & 49 & 14 & - \\
Hipertensão Arterial & 52 & 18 & 34 & - \\
Hipercolesterolemia & 29 & 15 & 13 & 01 \\
Dispnéia & 28 & 02 & 26 & - \\
Apnéia & 14 & - & 14 & 01 \\
Amenorréia & 08 & - & 07 & - \\
Broncoespasmo & 09 & 05 & 04 & 02 \\
Dermatopatia & 09 & 04 & 03 &
\end{tabular}




\section{DISCUSSÃO}

A incidência de obesidade e obesidade mórbida tem sofrido incrementos inegáveis tanto em países desenvolvidos, como em países em desenvolvimento ${ }^{7}$.

Embora a terapêutica inclua avanços progressivos no tratamento farmacológico, modificações no comportamento alimentar e incentivo à atividade física, freqüentemente têm-se constatado resultados insatisfatórios associados à redução da qualidade e da expectativa de vida ${ }^{8}$.

A cirurgia bariátrica é uma proposta terapêutica inovadora e recente para o problema, e vem realizando progressos e aperfeiçoamentos promissores.

Uma das cirurgias propostas para o tratamento da obesidade mórbida é a gastroplastia vertical com bandagem, descrita por Mason, que dominou o cenário bariátrico na década de $80^{\circ}$. No decorrer dos anos várias modificações foram associadas à cirurgia de Mason. Griffen ${ }^{10}$ introduziu a anastomose em Y-deRoux, que evita o refluxo bilio-pancreático e minimiza o risco de deiscência da anastomose. Alden ${ }^{11}$ iniciou o uso de grampeadores nas cirurgias restritivas, proporcionando maior facilidade e segurança. Torres ${ }^{12}$ introduziu a idéia de aumentar a extensão do intestino delgado ao se construir o Y-de-Roux, acrescentando um componente disabsortivo à operação antes restritiva, proporcionando uma redução ponderal maior.

Fobi $^{5}$ e Capella ${ }^{6}$ padronizaram operações que associam os princípios da gastroplastia vertical com bandagem de Mason ${ }^{9}$ aos das derivações gastrojejunais em Y-de-Roux, com adição de variantes técnicas, como; drenagem da cavidade abdominal e realização de gastrostomia no estômago excluso $^{13}$. Tendo em vista o risco de deiscências, Fobi $^{14}$ acredita que a drenagem preventiva junto à gastrojejunoanastomose identifica precocemente uma possível fístula, além de impedir a contaminação generalizada da cavidade e diminuir a necessidade de reoperação. A gastrostomia no estômago excluso permitiria realimentação precoce até que a deiscência cicatrize por segunda intenção. Com adoção desses cuidados adicionais, Fobi ${ }^{14}$ relata a ocorrência de fístulas em 2,6\% dos casos operados com esta técnica, não havendo necessidade de reoperação na maioria dos doentes.

A deiscência é a mais temida complicação pós-operatória precoce, com índices de 0 a $3 \%{ }^{15,16}$. Ela se dá tanto na anastomose quanto na linha de gram- pos. Sua profilaxia deve ser realizada ao máximo por várias táticas cirúrgicas, como; manipulação cuidadosa dos grampeadores, checagem da integridade da sutura por corantes e proteção complementar à linha de grampos. Não observamos deiscências de suturas, mas tivemos um caso de abscesso subfrênico periesplênico, tratado por punção guiada por tomografia, no $7^{\circ}$ dia de pós-operatório, que o exame radiológico da bolsa gástrica não evidenciou fístula. Fica a interrogação se não se trataria de pertuito fistuloso de baixo débito que ocorreu no pós-operatório imediato.

Nesta casuística, não realizamos drenagem abdominal e gastrostomia. No início, acreditávamos não haver necessidade de drenagem da cavidade pois a incidência de fístulas era baixa $(0,5-3,9 \%)$. Logo após, com a realização de um segundo plano invaginante, seromuscular, na bolsa gástrica, achamos que a chance de deiscência tornava-se menor ainda. Neste estudo, não houve ocorrência de deiscências ou fistulas anastomóticas ou na linha de grampeamento, fato que se deve, de acordo com nosso entendimento, ao uso de grampeadores com altura de grampo maior (carga verde) e pela invaginação da linha de sutura de grampos.Desta forma, não procedemos à drenagem rotineira da cavidade.

Dentre as complicações intra-operatórias mais comuns descritas na literatura, estão a gastroparesia, com índice de 0,5\%, acidentes com grampeamento, torção da alça de Roux interposta e lesão esplênica numa frequiência de $0,5-3,5 \%{ }^{15}$. Tivemos 10 casos de lesão esplênica, tratados em $60 \%$ dos casos com esplenectomia.

As obstruções intestinais são complicações comuns a todas laparotomias e podem ocorrer em qualquer época do pós-operatório. Fobi ${ }^{14}$ descreve, no seguimento dos seus doentes submetidos a GVBYR, ocorrência de 3,9\% de casos de obstrução intestinal por vários motivos: hérnia interna, bridas, aderências e outras. Nesta casuística somente dois casos tiveram esta complicação, devido à bridas, que foram corrigidas cirurgicamente.

As hemorragias internas são complicações incomuns no pós-operatório das GVBYR. Podem ser intraluminares ou intraperitoniais. Quando intraluminares se originam das linhas de sutura, lesões agudas ou úlceras pépticas ${ }^{17,18}$. Na nossa experiência ocorreram cinco casos de hemorragia digestiva alta causados por lesão aguda em um doente, sangramento na linha de sutura em um caso e outros 
três devido à úlcera de boca anastomótica. Todos tratados clinicamente e/ou endoscopicamente..

Nesta série tivemos 10 casos (4\%) de úlcera de boca anastomótica, apesar de todos os doentes serem submetidos à erradicação de Helicobacter Pylori e manterem uso de inibidor de bomba de prótons por dois meses no pós-operatório. A freqüência desta entidade após gastroplastia com bypass varia em torno de 0,1 à $20 \%{ }^{15,16,19}$.

Nas cirurgias bariátricas, o anel de silicone ou fita de polipropileno nos parece ter papel relevante na manutenção da perda de peso através do caráter restritivo que eles impõem ao reservatório gástrico ${ }^{20}$. Apesar de Capella ${ }^{6}$ ter ótimos resultados com a fita, nós utilizamos o anel de silicone pelo fato da fita com malha de polipropileno apresentar maior predisposição para extrusão e ser de mais difícil remoção quando esta complicação ocorre. As complicações relacionadas ao anel de silicone observadas nesta série foram migração em dois casos e extrusão em um doente $(1,2 \%)$. Essas complicaçõoes após GVBYR ocorreram em 1,8\% dos casos operados por Fobi ${ }^{14}$.

A colelitíase ocorre com freqüência após as operações de Fobi e Capella, e na quase totalidade dos operados por derivações bilio-pancreáticas. Isto ocorre devido à um supersaturação do colesterol na bile que é comum em pacientes em processo de emagrecimento. Neste estudo 31 doentes apresentaram litíase vesicular no pós-operatório,e foram submetidos à colecistectomia por laparotomia. O diagnóstico foi feito através de exame ultra-sonográfico realizado rotineiramente no pós-operatório. No pré-operatório todos eram submetidos à ultra-sonografia abdominal, apesar da obesidade ser fator limitante no diagnóstico ecográfico, e se constatado cálculo a colecistectomia era realizada no mesmo ato operatório. Assunto que merece questionamento é a realização da colecistectomia sincrônica à cirurgia bariátrica, dada a alta incidência de cálculos vesiculares no pós-operatório. No momento, os autores não praticam a colecistectomia preventiva.

Outra peculiaridade que merece comentário no pós-operatório da cirurgia bariátrica é a incisão cirúrgica. O seroma é a complicação mais comum após a GVBYR. Brolin ${ }^{15}$ relata incidência de $40 \%$ em sua experiência . Na nossa casuística realizamos drenagem a vácuo do tecido celular subcutâneo em todos os casos, com retirada do dreno por volta do $4^{\circ}$ dia de pós-operatório, quando a drenagem está abaixo de $20 \mathrm{ml}$. Tivemos três casos $(1,2 \%)$ de grandes seromas que mantiveram drenagem serosa pela cicatriz cirúrgica por vários dias, porém se resolveram somente com cuidados locais. Infecção de parede abdominal foi observada em três pacientes, sendo estes tratados com antibióticos e drenagem. As hérnias incisionais são relatadas na ordem de 5 à $16 \%{ }^{15,16}$. Neste estudo houve 16 casos $(6,4 \%)$.

As operações mistas como GVBYR, se não devidamente monitoradas acarretam deficiências de micro e macronutrientes. A desnutrição protêica se deve principalmente ao efeito restritivo do anel de silicone que dificulta a ingestão de carne vermelha e por isso os pacientes passam a evitá-la. $\mathrm{O}$ desvio do trânsito alimentar do duodeno, exclui a mais importante área de absorção do ferro e com isso sobrevêm a anemia ferropriva. Verificamos 68 casos de anemia ferropriva, dois casos de deficiência de vitamina B e um de desnutrição protêica.

Os pacientes submetidos a GVBYR apresentam no seu seguimento resultados muito gratificantes quanto à redução ponderal. Observa-se como regra geral, uma perda de peso em média de 35 a $40 \%$ do peso pré-operatório após 12 à 24 meses de cirurgia. Capella $^{21}$ na sua casuística, com "follow-up" de cinco anos, teve uma redução ponderal em torno de $40 \%$ do peso pré-operatório. Nossos resultados podem ser comparados com esses grupos, já que obtivemos uma redução percentual média de $36,5 \%$ no peso corporal, com um "follow-up" de no mínimo 12 meses. Tivemos dois casos $(0,8 \%)$ que apresentaram perda de peso menor que $30 \%$ após seguimento de um ano. Após 24 meses de cirurgia, existe tendência a uma discreta recuperação do peso na maioria dos casos, fato este ainda não observado por nós em virtude de um número insuficiente de pacientes nesta condição. A principal consequência da redução ponderal é uma grande melhora das doenças associadas à obesidade mórbida, e, por conseguinte na auto-estima e na qualidade de vida. Nosso seguimento ambulatorial demonstrou alta taxa de melhora ou cura das comorbidades, como ilustra a Tabela 4.

A taxa de mortalidade da GVBYR observada na série de Capella ${ }^{21}$ é de $0,3 \%$. Neste estudo não houve óbitos.

A cirurgia de obesidade, entre elas a GVBYR, apresenta na maioria das vezes evolução benigna, porém suas complicações quando ocorrem são catastróficas e exigem um alto grau de conhecimento técnico para seu diagnóstico. Embora a morbi-mortalidade pós-operatória se constitua num subproduto ine- 
vitável da experiência, a associação da capacidade técnica e harmonia multidisciplinar é indispensável para obtenção de melhores resultados.

De acordo com nossos resultados concluímos que a gastroplastia vertical com bandagem em
Y de Roux é efetiva em produzir perda de peso e se associa a uma baixa taxa de morbi-mortalidade. Baseamo-nos nessa experiência para recomendar a utilização deste procedimento para o tratamento de obesos mórbidos.

\begin{abstract}
Background: To analyze prospectively the results of patients submitted to vertical banded gastroplastyRoux-en-Y gastric bypass at the Hospital Universitário, Universidade Estadual de Londrina. Methods: We analized mortality, morbidity and weight loss of 250 consecutive patients with no previous bariatric surgery who were submitted to a combination of vertical banded gastroplasty and Roux-en-Y gastric bypass. Patients were followed up at least for one year. They had a mean body mass index of $46 \mathrm{Kg} / \mathrm{M} 2$. Results: The incidence of complications that required reoperation was $2 \%$. No deaths ocurred in the present study. After a follow up of one to three years we noticed an average decrease of $37.5 \%$ in the preoperative weight. In addition to the weight loss we detected a great improvement in the comorbidities and in some cases total control of the desease related to the obesity. Conclusions: Vertical banded gastroplastic Roux-en-Y gastric bypass is effective in promoting weight loss in morbid obese, and had a low mortality and morbidity.
\end{abstract}

Key Words: Gastroplasty; Obesity, morbid.

\section{REFERÊNCIAS}

1. Carmichael AR - Treatment for morbid obesity. Postgrad Med J, 1999, 75(879):7-12.

2. Alvarez-Cordero R - Final reflections: wellness after obesity surgery. World J Surg, 1998, 22(9):10181021.

3. Balsiger BM, Murr MM, Poggio JL, et al. - Bariatric surgery. Surgery for weight control in patients with morbid obesity. Med Clin North Am, 2000, 84(2):477489.

4. Mason EE, Tang S, Renquist KE, et al. - A decade of change in obesity surgery. National Bariatric Surgery Registry (NBSR) Contributors. Obes Surg, 1997, 7:189197.

5. Fobi MA, Lee - The surgical technique of the banded Roux-en-Y gastric bypass. J Obes Weight Regulation, 1989, 8:99-102.

6. Capella JF, Capella RF - Vertical banded gastroplastygastric bypass: preliminary report. Obes Surg, 1991, 1(4):389-395.

7. Heseker H, Schmid A-Epidemiology of obesity. Ther Umsch, 2000, 57(8): 478-481.

8. Seidell JC, Flegal KM - Assessing obesity: classification and epidemiology. Br Med Bull, 1997, 53(2):238-252.

9. Mason EE, Ito C - Gastric bypass in obesity. Surg Clin North Am, 1967, 47(6): 1345-1351.
10. Griffen WO, Young VL, Stevenson CC, et al. - Aprospective comparison of gastric and jejunoileal bypass procedures for morbid obesity. Ann Surg, 1977, 186(4):500-509.

11. Alden JF - Gastric and jejunoileal bypass. A comparison in the treatment of morbid obesity. Arch Surg, 1977, 112(7):799-804.

12. Torres JC, Oca C, Honer HM - Gastroplasty conversion to Roux-en-Y gastric bypass at the lesser curvature due to weight loss failure. Am Surg, 1985, 51(10):559-562.

13. Berti LV, Oliveira MR, Elias AA, et al. - Gastrostomia e Drenagem da cavidade ( GSDR) nas GVYRA. Bol Cir Obesidade, 2001, 2(4):25.

14. Fobi MA, Lee H, et al. - "Transected silastic ring vertical gastric bypass with jejunal interposition, a gastrostomy and a gastrostomy site marker (Fobi pouch operation for obesity)." In Dietel M, Cowan GSM (eds) - Update: Surgery for the morbidly obese patient. Toronto. FD Comunications, 2000.

15. Brolin R - "Complications of surgery for severe obesity". In Sugerman H, Sooper N (eds) - Problems in General Surgery. Philadelfia, 2000.

16. Byrne TK - Complications of surgery for obesity. Surg Clin North Am, 2001, 81(5):1181-1193.

17. Brolin RE-Healing of the stapled stomach in bariatric operations. Surgery, 1993, 113(5):484-490.

18. Flickinger EG, Pories WJ, Meelheim HD, et al. - The Greenville gastric bypass. Progress report at 3 years. Ann Surg, 1984, 199(5):555-562. 
19. Fobi M, Lee H, Igwe D, et al. - Band erosion: incidence, ethiology, management and outcome after banded vertical gastric bypass. Obes Surg, 2001, 11(6):699707.

20. Silva LGO, Barroso FL, Zucaro AM, et al. - Análise da incidência e do significado de úlceras e erosões após gastroplastia com anel de silicone e bypass: estudo em 168 pacientes. Bol Cir Obesidade, 2001, 2(4):25.
21. Capella JF, Capella RF - Gastro-gastric fistulas and marginal ulcers in gastric bypass procedures for weight reduction. Obes Surg, 1999, 9(1):22-28.

Endereço para correspondência :

Antonio Carlos Valezi

Rua Santos, n ${ }^{\circ} 777$; Apto: 1302

CEP: 86020-021 - Londrina - PR

E-mail: valezi@sercomtel.com.br 